\title{
La Universidad Pública y su rol en la pandemia COVID-19: laboratorios de diagnóstico al servicio de la red nacional de laboratorios de influenza y otros virus respiratorios
}

Rosana Toro 1; Juan Manuel Unzaga 2; Gonzalo Márquez 3; Carlos Javier Panei 2; Alejandra Bosch

1; Guillermo Docena 1; Martín Carlos Abba 4; Daniela Hozbor "; Equipo Red de Laboratorios de la UNLP

${ }^{1}$ Facultad de Ciencias Exactas - UNLP; ${ }^{2}$ Facultad de Ciencias Veterinarias - UNLP; ${ }^{3}$ Presidencia de la UNLP; ${ }^{4}$ Facultad de Ciencias Médicas - UNLP; rosanat26@hotmail.com

Resumen: La enfermedad por coronavirus 2019 (COVID-19) se informó por primera vez en Wuhan, China, el 31 de diciembre de 2019, declarándose emergencia de salud pública de importancia internacional en primera instancia y posteriormente elevada a la categoría de pandemia. Ante la grave situación sanitaria producto de la propagación del SARS-CoV-2, el Ministerio de Salud de la Provincia de Buenos Aires y la Universidad Nacional de La Plata establecieron un convenio de cooperación con la finalidad de que la Universidad Nacional de La Plata provea capacidad diagnóstica al sistema sanitario. Luego de un período de adecuación de los laboratorios e implementación de protocolos se inició tempranamente con el diagnóstico del SARS-CoV-2, habiendo procesado a fines de septiembre de 2020 más de 32.000 muestras provenientes de diversos Municipios de las Regiones Sanitarias I, II, IV, VI, X Y XI de la Provincia de Buenos Aires. Los laboratorios participantes de las Facultades de Ciencias Exactas, Ciencias Médicas y Ciencias Veterinarias forman parte de los Laboratorios de referencia de COVID-19 de la Provincia de Buenos Aires y articulan dicha labor con diversas actividades de investigación, docencia y desarrollo con la finalidad de proveer soluciones al sistema de salud en el contexto de la pandemia.

Palabras clave: diagnóstico; SARS-CoV2; COVID-19

Recibido: 10/10/2020 Aceptado: 13/10/2020

DOI: https://doi.org/10.24215/26838559e015 
The Public University and its role in the COVID-19 pandemic: diagnostic laboratories at the service of the national network of influenza and other respiratory viruses

Abstract. Coronavirus 2019 (COVID-19) was initially reported in Wuhan, China, on December 31 2019, and later declared as public health of international relevance and pandemic disease. Given the serious health situation resulting from the spread of SARS-CoV-2, the Ministry of Health of the Province of Buenos Aires and the Universidad Nacional de La Plata established a cooperation agreement to provide the diagnostic capability to the Public Health System. After an implementation period, Universidad Nacional de La Plata based labs began early with the diagnosis of SARS-CoV-2, having processed more than 32,000 samples derived from Sanitary Regions I, II, IV, VI, X, and XI of the Province of Buenos Aires. The participating laboratories at UNLP are part of the Reference Laboratory for COVID-19 of the Province of Buenos Aires and participate in research, teaching, and development activities to provide solutions to the Public Health System in the context of this pandemic disease.

Key words: diagnosis; SARS-CoV2; COVID-19

\section{Equipo Red de Laboratorios de la UNLP}

\begin{tabular}{|c|c|}
\hline Alejandro Aiello ${ }^{4}$ & Nadia Analía Fuentealba ${ }^{2}$ \\
\hline Pablo Martín Aispuro ${ }^{1}$ & Magalí Gabrielli ${ }^{1}$ \\
\hline Nicolás Ambrosis ${ }^{1}$ & Horacio Garda ${ }^{4}$ \\
\hline Andrés Angelletti ${ }^{1}$ & Alejandra Giordano ${ }^{1}$ \\
\hline Melina Anello ${ }^{1}$ & Solange Giordano ${ }^{1}$ \\
\hline Lucía Argenio ${ }^{2}$ & Carlos Golijow $^{2}$ \\
\hline Carolina Gabriela Aspitia ${ }^{2}$ & María Gonzalez Baro 4 \\
\hline Keila Belhart ${ }^{1}$ & Carolina Jaquenod De \\
\hline Cecilia Bergna $^{1}$ & \\
\hline Flavio Blanco $^{1}$ & Mauricio Kraemer ${ }^{4}$ \\
\hline Daniela Bottero ${ }^{1}$ & Antonio Lagares ${ }^{1}$ \\
\hline María Emilia Bravi ${ }^{2}$ & Yanina Lamberti ${ }^{1}$ \\
\hline Victoria Cabassi ${ }^{1}$ & Sabrina M.L. Lavarías ${ }^{4}$ \\
\hline & Aníbal Lodeiro ${ }^{1}$ \\
\hline
\end{tabular}

Alejandro Orlowski 4 Marcelo Italo Pecoraro ${ }^{2}$

Nicolás Pedrini ${ }^{4}$

Erica Pereyra ${ }^{4}$

Lisandro Petraglia ${ }^{1}$

Jimena Quintana Corvalan ${ }^{1}$

Magdalena Rambeaud ${ }^{2}$

Mauricio Reynoso ${ }^{1}$

María Eugenia Rodríguez ${ }^{1}$

Víctor Romanowsky ${ }^{1}$

Gabriela Rudd ${ }^{2}$

Erika Rudi ${ }^{1}$

Martín Rumbo ${ }^{1}$ 
Innovación y Desarrollo Tecnológico y Social (2020) 2 (2): 25-50- Número especial COVID-19

Romina Canzoneri ${ }^{4}$
Paula Carasi ${ }^{1}$
Noelia Costantini $^{4}$
Andrés Cordero $^{1}$
María Virginia Croce ${ }^{4}$
Laura Delaplace $^{1}$
Sebastián García
Einschlag $^{1}$
Ulises Fleitas ${ }^{1}$
Flavio Francini ${ }^{4}$

Luciano Malaissi ${ }^{1}$

Flavia Mazzini ${ }^{1}$

María Victoria Mencucci ${ }^{4}$

Mauro Montanaro ${ }^{4}$

Verónica Moscoso ${ }^{4}$

Gastón Andrés Moré 2

Victoria Nadalich ${ }^{1}$

Maximiliano Orezzo ${ }^{1}$

Javier Origlia ${ }^{2}$

\author{
Marianela Santana ${ }^{4}$ \\ Lorena Tau $^{1}$ \\ Carina Tersigni ${ }^{1}$ \\ Marco Tizzano ${ }^{2}$ \\ Soledad Traubenik ${ }^{1}$ \\ Hugo Valdez ${ }^{1}$ \\ Florencia Ventura ${ }^{1}$ \\ Martín Vila Petroff 4 \\ Eugenia Zurita ${ }^{1}$
}

\section{Contexto de la implementación de la capacidad diagnóstica}

Las enfermedades emergentes y re- emergentes presentan un desafío en los sistemas de salud pública. La aparición de un nuevo coronavirus humano en diciembre del 2019 en la ciudad de Wuhan, China, desencadenó una colaboración internacional que permitió identificar y secuenciar el nuevo virus y diseñar un test diagnóstico en poco menos de un mes (Wu et al, 2020; Na et al, 2019; Corman et al, 2020). El 30 de enero de 2020 la Organización Mundial de la Salud declaró el brote del nuevo coronavirus como una emergencia de salud pública de importancia internacional y posteriormente la elevó a la categoría de pandemia. (https://www.who.int/home). En Argentina, el Ministerio de Salud de Nación diseñó diferentes estrategias de atención y cuidado de pacientes con enfermedad por SARS CoV 2 (COVID-19), implementando el diagnóstico por PCR en laboratorios de la Red 
Nacional de Influenza y otros virus respiratorios, a los que sumó nuevos laboratorios con el fin de aumentar la capacidad de respuesta diagnóstica.

El 18 de marzo del 2020, el Presidente de la UNLP, Dr. Fernando Tauber, puso a disposición del Ministro de Salud de la Provincia de Buenos Aires las capacidades existentes en las distintas Facultades, para el eventual procesamiento y diagnóstico del SARS-CoV-2. Luego de la declaración del Aislamiento Social Preventivo y Obligatorio (ASPO) por parte del Gobierno Nacional en marzo del corriente año (DECNU-2020-297-APN-PTE), el Ministerio de Salud de la Provincia de Buenos Aires concretó junto con la UNLP el inicio del trabajo en colaboración para el diagnóstico molecular de SARS-CoV-2, en apoyo a la Red Nacional de Laboratorios de Influenza y otros virus respiratorios a través de laboratorios de las Facultades de Ciencias Exactas, Ciencias Médicas y Ciencias Veterinarias en el marco del convenio específico (CONVE-2020-06345305-GDEBA-MSALGP). En este sentido, se conformó un Comité Técnico integrado por representantes de la universidad y de las 3 facultades intervinientes, con el fin de conformar los grupos de trabajo integrados por docentes-investigadores y la puesta en marcha y ejecución de los laboratorios. Dicho proceso implicó, la implementación de normas de bioseguridad, arreglos edilicios, validación del equipamiento existente, la adquisición de nuevo equipamiento, la capacitación del personal y la redacción de los protocolos operativos para el correcto funcionamiento de los laboratorios. 


\section{Grado de relevancia y pertinencia}

La vigilancia epidemiológica en la actual situación consiste en detectar de manera temprana casos, permitiendo la atención adecuada de los pacientes y la implementación de las medidas de investigación, prevención y control tendientes a reducir el riesgo de diseminación de la infección en la población, así como la adecuada atención de los casos graves. Para ello, es imprescindible contar con un diagnóstico etiológico rápido y eficaz que sea el punto de partida para un correcto manejo epidemiológico de la pandemia. Así, la vigilancia de COVID-19 se inscribe dentro de las infecciones respiratorias agudas (ETI, bronquiolitis, neumonía, IRAG, IRAGI y sospecha Virus emergente) que incluye los servicios de atención de los distintos subsectores, las áreas de epidemiología municipales, regionales, provincial y nacional, la red nacional de laboratorios de influenza y otros virus respiratorios, laboratorios privados con capacidad para el diagnóstico y los laboratorios nacionales dependientes de la ANLIS, entre otros. En este sentido, la conformación de los laboratorios de la UNLP en apoyo directo a los citados laboratorios, se suma a la acción en ayuda colaborativa al diagnóstico de COVID-19 en el territorio provincial, atendiendo en forma temprana la vasta demanda existente y en cumplimiento directo con uno de los pilares que prevé el Estatuto de la UNLP, en relación a la integración de la Universidad en tanto institución del Estado Nacional con la comunidad de la cual forma parte, promoviendo a contribuir en la resolución de las problemáticas sociales/sanitarias existentes. Debido a que las Facultades de 
Ciencias Exactas, Ciencias Médicas y Ciencias Veterinarias cuentan con laboratorios con las condiciones edilicias y de equipamiento correspondiente a los niveles de bioseguridad 2 (BSL2), así como con profesionales adiestrados y con experiencia en el área, se constituyeron en unidades académicas de referencia para la implementación de los procedimientos de diagnóstico.

\section{Demanda de capacidad diagnóstica del SARS-CoV-2}

La actual pandemia requiere que, en relación a la vigilancia epidemiológica, se aúnen todos los esfuerzos posibles para dar respuesta inmediata y certera para asegurar la contención sanitaria que la población requiere. En este sentido, desde que el 3 de marzo del corriente año se confirmó el primer caso de COVID-19 en Argentina, correspondiente a un viajero proveniente de Italia, la demanda en cuanto al diagnóstico molecular de SARS-CoV-2 ha ido en aumento, sobre todo en relación a las políticas públicas desarrolladas, tal es el caso del Dispositivo Estratégico de Testeo para Coronavirus en Territorio de Argentina (DETeCTAr). En este mismo sentido, la Universidad Pública, como parte integrante del Estado, ha demostrado estar a la altura de las necesidades respondiendo en forma rápida a la demanda que desde la Red Nacional de Laboratorios de influenza y otros virus respiratorios se visualizó respecto al aumento del diagnóstico de COVID-19 en el territorio de la Provincia de Buenos Aires. 
Innovación y Desarrollo Tecnológico y Social (2020) 2 (2): 25-50- Número especial COVID-19

Implementación de una red de laboratorios para la detección del SARS-CoV-2 en unidades académicas de la UNLP

\section{Conformación de un Comité Técnico para el Diagnóstico de SARS-CoV-2}

En base a un relevamiento interno realizado por la Presidencia de la UNLP con el objetivo de magnificar las capacidades internas para la realización de diagnósticos de esta enfermedad, el 23 de marzo de 2020 se procede a constituir un Comité Técnico coordinado por la Presidencia y con representación de las Facultades de Ciencias Exactas, Ciencias Médicas y Ciencias Veterinarias. La finalidad del mismo fue la de coordinar las líneas de acción entre unidades académicas, en lo que respecta al diagnóstico del SARS-CoV-2. Las primeras actividades realizadas fueron las relacionadas con la puesta a punto de los laboratorios, en las cuales fue clave el diálogo y la puesta en valor de la experiencia previa de los diversos actores involucrados. En este sentido, cabe destacar el rol central de dicho comité en el desarrollo de las acciones llevadas adelante por la UNLP para acompañar al sistema de diagnóstico provincial. El trabajo del mismo permitió no sólo promover actividades de cooperación entre laboratorios, sino también la coordinación de todas las acciones de logística y provisión de insumos entre el Ministerio de Salud de la Provincia y las facultades de la UNLP involucradas. De esta manera el funcionamiento del comité ha resultado fundamental para generar debates, articular tareas y diseñar estrategias para dar respuesta a las demandas sanitarias. 
A continuación, se describen el proceso de implementación y las actividades de diagnóstico que se desarrollan en las tres unidades académicas.

\section{Facultad de Ciencias Exactas:}

\section{Laboratorio de Salud Pública}

El Laboratorio de Salud Pública (LSP) de la Facultad de Ciencias Exactas, como parte del Programa de Salud de la Secretaría de Extensión de dicha Unidad Académica, desarrolla desde hace más de 10 años una intensa actividad de diagnóstico bioquímico y de enfermedades infecciosas. Además, desde su creación lleva a cabo una intensa actividad docente con alumnos de las Prácticas Bioquímicas y con Extensionistas quienes se integran en actividades en los distintos sectores, posicionando al LSP como una unidad de docencia-extensión e investigación del Área Bioquímica Clínica. En el año 2009 debido a la pandemia de Gripe (Influenza H1N1) se formó en el LSP un equipo de trabajo en diagnóstico viral, capacitado en el Instituto Malbrán para dar respuesta a dicha emergencia. Como resultado se realizaron diagnósticos de Influenza A y $B$, y se llevaron a cabo actividades de docencia e investigación en colaboración con el Servicio de Virus Respiratorios del Instituto Malbrán a cargo de la Dra. Elsa Baumeister, docente de Virología Clínica de nuestra Facultad. En el año 2014 el laboratorio de virología del LSP ha creado el Centro de asesoramiento, prevención y testeo de VIH, Hepatitis Virales y Sífilis, que desde entonces realiza jornadas de testeo y prevención de infecciones de 
transmisión sexual destinados tanto a la comunidad universitaria como a la población general.

El objetivo final de todas las actividades desarrolladas por el LSP apunta a mejorar la formación profesional de nuestros graduados, incentivando el compromiso con el trabajo territorial y en los diversos ámbitos académicos y de Salud Pública.

Ante la emergencia del nuevo Coronavirus, en el mes de marzo de 2020 la Facultad de Ciencias Exactas decidió sumar capacidades y equipamiento de distintos centros de investigación para apoyar al LSP en la implementación de metodologías moleculares de identificación molecular de SARS-CoV-2, en hisopado de pacientes obtenidos de las diferentes Regiones Sanitarias de la Provincia de Buenos Aires.

El primer desafío fue organizar el trabajo para articular con los niveles de atención hospitalarios y las autoridades de los Ministerios de Salud Provincial y Nacional. Fue importante en este sentido, la experiencia con que contaba el LSP para llevar a cabo el procesamiento general de muestras clínicas, desde la recepción de las mismas hasta el diagnóstico molecular y/o serológico. Los profesionales docentes del laboratorio de virología recibieron el entrenamiento del Instituto Malbrán para la implementación de kits de diagnóstico, integrando la Red Nacional de Diagnóstico de SARS-CoV-2 colaborando con el Sistema de Salud en el monitoreo y manejo de la pandemia. El equipo de trabajo, recibió capacitación por parte de la Pro-secretaría de Hábitat y Espacios y la Dirección de Seguridad, Higiene y Desarrollo Sustentable de nuestra facultad para el uso del equipo de protección personal y sobre prácticas 
de bioseguridad. Con el apoyo de las autoridades de la facultad y la UNLP, se construyó un espacio para la recepción de muestras clínicas patogénicas, que garantiza hoy la seguridad de las personas que trabajaran en ello.

Desde el inicio de las actividades, articularon con el LSP los distintos grupos de docentes, investigadores y alumnos de nuestra facultad constituyéndose un grupo interdisciplinario de trabajo con los distintos centros de investigación de la facultad, CONICET y el LSP. Se conformó así el Grupo COVID Exactas, constituido por docentes, investigadores, becarios y estudiantes de distintos Institutos de la facultad como el Instituto de Estudios Inmunológicos y Fisiopatológicos (IIFP), el Instituto de Biotecnología y Biología Molecular (IBBM), el Instituto de Investigaciones Fisicoquímicas Teóricas y Aplicadas (INIFTA), y el Centro de Investigación y Desarrollo en Fermentaciones Industriales (CINDEFI). El nuevo Laboratorio de Virología se creó entonces sumando las capacidades, recursos humanos y equipamiento del Grupo COVID Exactas, de otros Programas de Extensión de nuestra facultad, de voluntarios, de investigadores y bioquímicos provenientes de hospitales con capacitación técnica en Biología Molecular. Con el esfuerzo de nuestra facultad y la Universidad se incorporaron nuevos cargos docentes que permitieron poner en marcha un gran equipo de trabajo, que cuenta hoy con más de 30 personas entre docentes, investigadores y alumnos. A través del sector de Gestión de Calidad del LSP y la División de Redes de Internet de nuestra facultad se logró incorporar un sistema informático de laboratorio que garantiza la trazabilidad 
de las muestras asegurando la calidad del trabajo desde la recepción de las mismas hasta la obtención del informe final. Es importante resaltar además que el LSP se incorporó al proyecto en red de secuenciación de material genómico de SARS-CoV2 "Genómica de los virus SARS-CoV-2 productores de COVID-19 en Argentina. Análisis integral de aspectos genéticos, clínicos y evolutivos de cepas autóctonas y su impacto en el diagnóstico y la epidemiología local y global".

Desde el 27 de abril hasta fin de septiembre, el LSP ha procesado más de 16.000 muestras provenientes principalmente de las regiones sanitarias $\mathrm{VI}, \mathrm{X}$ y XI. La capacidad de procesamiento se estimó inicialmente en 100 muestras diarias, pero la demanda aumentó llegando a un máximo de casi 400 muestras en el mes de Julio. El poder responder a esta tremenda demanda fue gracias al compromiso de cada integrante del equipo de trabajo y a la organización del LSP en los distintos niveles: recepción de muestras, preparación y procesamiento, lectura e interpretación de resultados, informe final de resultados en el Sistema Integrado de Información Sanitario Argentino (SISA) y comunicación con los médicos y bioquímicos del nivel de atención hospitalaria.

El grupo de trabajo del LSP articula con el grupo COVID Exactas, el laboratorio VacSal y con las Brigadas Sanitarias “Ramona Medina” de nuestra Facultad. Recientemente el Grupo COVID Exactas recibió financiamiento del MINCYT en el marco del proyecto "Fortalecimiento de las capacidades de diagnóstico e investigación del Laboratorio de Salud Pública de la Facultad de Ciencias Exactas (UNLP)". Estas interacciones 
multidisciplinarias impulsaron nuevos proyectos de investigación en 3 ejes de trabajo: diagnóstico (RT-qPCR sobre muestras de hisopados de pacientes), vigilancia (análisis en "pooles" de muestras de hisopados por RT-qPCR y anticuerpos por serología) y validación y desarrollo de métodos analíticos (biología molecular, detección de antígenos virales y de anticuerpos, detección de marcadores de vigilancia en orina por métodos espectroscópicos).

En relación a las actividades de vigilancia epidemiológica, el LSP ha formado parte del equipo de trabajo conformado por profesionales de diferentes Unidades de Pronta Atención (UPA) de La Plata, bioquímicos coordinados desde el Colegio de Bioquímicos de la Provincia de Buenos Aires y los brigadistas de la Facultad de Ciencias Exactas. Este grupo, integrado por aproximadamente 150 personas, se ha dedicado a monitorear distintos barrios de La Plata. En particular, el LSP ha participado en la realización de las extracciones de sangre y la detección de anticuerpos específicos de SARS-CoV-2 por ELISA con el kit COVIDAR (aportado por el Ministerio de Salud). A su vez esta actividad ha sido coordinada con el Instituto de Hemoterapia de La Plata donde se realizan las donaciones de plasma de convalecientes. La actividad desarrollada en pocos meses queda plasmada en la creación del nuevo laboratorio de virología dentro del LSP que combina diagnóstico clínico, docencia e investigación, para responder hoy a las necesidades sanitarias de esta pandemia de COVID 19, con el fin de permanecer en la post-pandemia para responder a diferentes requerimientos sanitarios asociados a infecciones virales. 


\section{Laboratorio VacSal del Instituto de Biotecnología y Biología Molecular (FCE-}

\section{UNLP CONICET)}

El laboratorio VacSal además de las actividades de investigación en el área de Vacunología Humana y de interacción huésped-patógeno es Centro de Referencia de la patología denominada pertussis o tos convulsa, una enfermedad respiratoria de origen bacteriano resurgente. El laboratorio ha participado activamente desde hace más de 22 años en la vigilancia laboratorial de pertussis, realizando actividades de diagnóstico, capacitación y transferencia de metodologías a nivel nacional e internacional (Ministerio de Salud, 2010; Falleiros Arlant, 2014). Ha contribuido de manera sustancial al armado de la red de vigilancia laboratorial para pertussis. Con esta experiencia y en respuesta a la situación de pandemia causada por el virus reemergente SARS-CoV-2, el laboratorio se puso a disposición de la Facultad de Ciencias Exactas y de las autoridades sanitarias para contribuir en dar respuesta a la situación de crisis sanitaria que estamos atravesando. A fines de mayo, en forma articulada con el Ministerio de Salud de la Provincia de Buenos Aires, comenzamos con las actividades de implementación, validación de equipos y kits y puesta a punto de la detección de SARS-CoV-2 a través de la estrategia de pooles o agrupamiento de muestras clínicas. Este trabajo surgió además de la interacción con docentesinvestigadores de la Universidad de Buenos Aires, en particular con el grupo dirigido por los Dres. Roberto Etchenique y Adali Pecci. Conformamos primeramente el 
equipo de trabajo, que en la actualidad está constituido por docentesinvestigadores, becarios y profesionales de apoyo en un total de 10 y ajustamos los protocolos de trabajo para COVID-19. Trabajamos entonces en la evaluación de la factibilidad de realizar agrupamiento de muestras clínicas antes de proceder a la extracción de ARN lo cual posibilitaría no solo el ahorro en kits de RT-qPCR sino además los de la extracción de ARN. Establecimos y pusimos a punto todas las condiciones de trabajo, y una vez finalizada la etapa realizamos una prueba piloto trabajando en forma paralela con la estrategia de pooles y la detección individual a partir de un mismo set de muestras. Una vez validada la estrategia, y en respuesta al diseño de vigilancia activa diseñada por Ministerio de Salud de la Provincia de Buenos Aires, se comenzó a recibir muestras clínicas provenientes de individuos asintomáticos u oligosintomáticos (incluyendo residentes, personal de salud, otros trabajadores) que asisten a lugares semicerrados como geriátricos, hogares de asistencia a personas, clínicas de salud mental, comisarías, penitenciarías, de distintas localidades de la provincia de Buenos Aires. La vigilancia activa busca así detectar tempranamente individuos infectados de forma de posibilitar la rápida implementación de acciones sobre el individuo y su entorno (Provincia de Buenos Aires, 2020).

Desde el 28 de mayo a finales de septiembre hemos procesado más de 8.000 muestras clínicas provenientes de localidades de las regiones sanitarias I, II, IV, VI, X y XI. Las muestras a procesar en nuestro laboratorio son recepcionadas por el LSP 
utilizando el circuito por ellos diseñado (ver arriba). Para el procesamiento de las muestras en el laboratorio se destinan 3 días de trabajo de forma de dejar los otros días para apertura de pooles, evaluación de kits y de otras estrategias posibles de ser implementadas. Los resultados se informan a los efectores de salud y a SISA dentro de las 24-48 hs de recepcionada la muestra en VacSal. Semanalmente recibimos un promedio de 600 muestras, pero este número es muy variable. Hasta la fecha el máximo número de muestras procesadas en un día fueron 438. Es de destacar que el ahorro en kits de esta estrategia es dependiente de la prevalencia de casos positivos. Si bien la positividad ha cambiado desde que comenzamos el trabajo en COVID-19, el promedio de ahorro es del 66\%; dicho de otro modo, con un kit podemos evaluar la presencia de SARS-CoV-2 en tres individuos, lo cual permite una mayor velocidad y amplitud en el testeo de asintomáticos y oligosintomáticos. En las últimas semanas del mes de septiembre además de trabajar con la estrategia de pooles, realizamos análisis de muestras clínicas individuales ya que provienen de pacientes cuya sintomatología cumple con la definición clínica de COVID-19. Recibimos así muestras de Bahía Blanca, Pergamino y Ezeiza. Además de las actividades de laboratorio, hemos contribuido en el diseño de instructivos y de las fichas epidemiológicas que se emplean en la estrategia de pooles. Hemos transferido la metodología a otras instituciones y hemos realizado actividades de asesoramiento de la implementación de dicha estrategia. 


\section{Facultad de Ciencias Médicas}

El 25 de marzo de 2020 la Facultad de Ciencias Médicas procedió a constituir una red de laboratorios (denominada Redlab FCM-UNLP) integrada por el Instituto de Investigaciones Bioquímicas de La Plata (INIBIOLP), el Centro de Investigaciones Cardiovasculares $(\mathrm{CIC})$, el Centro de Investigaciones Inmunológicas Básicas y Aplicadas (CINIBA) y el Centro de Endocrinología Experimental y Aplicada (CENEXA). Dicha red se constituyó en función de un relevamiento de sus capacidades en cuanto a la disponibilidad de cabinas de bioseguridad de tipo 2, equipos de PCR de tiempo real y la disponibilidad de voluntarios con experiencia en biología molecular y en el manejo de agentes infecciosos. De esta manera se conformó un equipo de trabajo compuesto por 18 profesionales Bioquímicos, Biotecnólogos y Biólogos Moleculares (Docentes de UNLP e Investigadores y CPA del CONICET) con el objetivo de acondicionar los laboratorios, definir la logística de las muestras y definir los protocolos de trabajo. El proceso de reacondicionamiento de los laboratorios en lo que respecta a bioseguridad, validación de cabinas de seguridad, recepción y logística de muestras fue supervisado por la Secretaria de Bioseguridad e Higiene de la UNLP. Brevemente, se constituyeron equipos de trabajo distribuidos en los sectores de: 1) Recepción de muestras, 2) Procesamiento y extracción de ARN, 3) Análisis por PCR de tiempo real y 4) Carga de resultados al SISA. Los cuatro sectores se encuentran conectados a un sistema integrado de carga de datos que facilita la trazabilidad de las muestras en tiempo real y facilita la coordinación de los grupos 
de trabajo. En la recepción se procede al ingreso de las muestras al sistema, se digitalizan las fichas epidemiológicas y se labra un acta de recepción de manera diaria. Desde el inicio de las actividades (26 de mayo de 2020) hasta finales de septiembre se procesaron más de 4.500 muestras. Actualmente se procesan 350 muestras semanales (de lunes a sábado) provenientes de diversas Instituciones del sector Público y Privado de la Región Sanitaria XI (SAME La Plata, Hospital Municipal Francisco Caram Brandsen, Instituto Médico de Brandsen, Hospital Zonal Especializado Dr. Noel H. Sbarra, Nueva Clínica del Niño de La Plata S.R.L., Hospital Interzonal de Agudos Especializado en Pediatría Sor María Ludovica, etc.).

Las muestras recepcionadas corresponden principalmente a hisopados nasofaríngeos y aspirados traqueales que se procesan mediante el empleo de columnas de purificación de ARN (Viral Nucleic Acid Extraction Kit II, GeneAid) o mediante el sistema de beads magnéticas (MGIEasy Nucleic Acid Extraction Kit). La detección del ARN del SARS-CoV-2 se realiza mediante el empleo de sistemas de qPCR Taqman (GeneFinder COVID-19 Plus RealAmp Kit, BGI Real-Time Fluorescent RT-PCR Kit for Detecting SARS-CoV-2, DisCoVery AP-Biotech) provistos por el Ministerio de Salud de la Provincia de Buenos Aires. Los resultados se informan a los efectores de salud y a SISA dentro de las $12-24$ hs. de recepcionada la muestra. Es importante mencionar que dichas actividades dieron lugar y articulan con actividades de investigación y desarrollo en el marco de un proyecto financiado Programa de Articulación y Fortalecimiento Federal de las Capacidades en Ciencia y 
Tecnología COVID-19 del Ministerio de Ciencia, Tecnología e Innovación de la Nación (MINCyT). El eje central del proyecto consiste en el desarrollo de un sistema RT-qPCR para la determinación de la carga viral del SARS-CoV-2 a partir de hisopados nasofaríngeos en pacientes con diagnóstico confirmado de COVID-19. En primer lugar, dicho desarrollo se pondrá a disposición del sistema sanitario provincial y nacional a la manera de un servicio tecnológico brindado por la Facultad de Ciencias Médicas de la UNLP. En segundo lugar, se evaluará la posibilidad de producción y distribución del sistema de detección de carga viral a todo laboratorio de salud pública que desee evaluarlo previa autorización y certificación por las instituciones involucradas. Además, dicha metodología se utilizará como herramienta para estudiar la relación entre la carga viral y el índice de disfunción pulmonar y marcadores de fase aguda al momento de la internación del paciente y luego de 7 días de su hospitalización.

\section{Facultad de Ciencias Veterinarias}

En un primer momento se pusieron en marcha dos ejes fundamentales para cumplir con el funcionamiento de la Unidad de Diagnóstico COVID-19 FCV-UNLP, por un lado (a) se conformó el grupo de trabajo y por otro; (b) se evaluó, seleccionó el laboratorio y se realizaron los arreglos edilicios y la validación y compra de equipamiento necesario para el buen desempeño de las actividades correspondientes. 
(a) El grupo de trabajo está integrado por 13 docentes-investigadores (UNLPCONICET) pertenecientes al Instituto de Genética Veterinaria (IGEVET), Laboratorio de Inmunoparasitología (LAINPA) y Laboratorio de Virología (LAVIR) de las carreras de Medicina Veterinaria y Microbiología Clínica e Industrial de la FCV-UNLP. Respecto a su formación académica, los participantes constituyen un grupo heterogéneo y multidisciplinar integrado por Médicos Veterinarios, Bacteriólogos y/o Microbiólogos, Lic. en Biología y Biotecnólogos que voluntariamente participaron de 3 encuentros virtuales para la puesta en conocimiento, selección y diseño de los equipos de trabajo que, de acuerdo a las normativas de bioseguridad vigentes, están conformados por 3 subgrupos de 4 integrantes cada uno que desarrollan el trabajo en días definidos de lunes a viernes. Para la ejecución de las tareas, cada uno de los subgrupos cuenta con profesionales dedicados a tareas específicas (1-recepción de muestras y asistencia general, 2- extracción de ARN en cabina, 3- real time RT-PCR y pre-informes y 4-informe final al SISA). Previo a la puesta en marcha los profesionales seleccionados recibieron capacitaciones técnico-científicas específicas como así también una capacitación dictada por la Dirección de Seguridad, Higiene y Desarrollo Sustentable-UNLP para brindar los conocimientos necesarios para el desarrollo bio-seguro del diagnóstico y la disposición de residuos.

Por otro lado, y como medida de control del personal abocado al diagnóstico, a cada integrante del laboratorio se le realiza un hisopado orofaríngeo con una frecuencia 
mensual. Las muestras de ARN obtenidas de cada uno son procesadas por real time $R T$-PCR formando pooles por cada subgrupo de trabajo.

(b) La Facultad de Ciencias Veterinarias, acordó con los representantes del Ministerio de Salud de la Provincia de Buenos Aires, la recepción y procesamiento de muestras provenientes del monitoreo llevado a cabo en barrios populares como parte del DETeCTAr, principalmente. Para ello, en el mes de mayo se conformó la Unidad de Diagnóstico de COVID-19, que funciona en las instalaciones de la Cátedra de Tecnología y Sanidad de los Alimentos, laboratorio preexistente que fue seleccionado bajo el asesoramiento de personal perteneciente a la Dirección de Seguridad, Higiene y Desarrollo Sustentable-UNLP. En este sentido, el laboratorio fue acondicionado acorde a las normas de bioseguridad requeridas para llevar a cabo el diagnóstico de SARS-CoV-2. Se realizaron modificaciones edilicias para la recepción de muestras y se acondicionaron los espacios de circulación y señalética dentro del predio de la facultad para una correcta ubicación y transporte de la muestra por parte de los ambulancieros destinados a la tarea. Asimismo, se establecieron protocolos de circulación e ingreso al laboratorio, limpieza y desinfección del mismo, uso de elementos de protección personal (EPP), ingreso de muestras, toma de muestras, extracción de ARN y amplificación por real time RTPCR. Se procedió a la validación (Empresa Validaciones Absolutas) de la cabina de seguridad de tipo II (Sabella, Argentina), se reubicó para su uso el equipo MIC 
qCycler que pertenece al LAINPA y se adquirió el programa LabWin (Biodata) para la gestión técnica y administrativa integral de los datos.

Actividad diaria del laboratorio: se reciben principalmente muestras de personas residentes en alguno de los municipios que componen la Región Sanitaria VI y XI, como así también muestras provenientes de establecimientos sanitarios privados de la ciudad de La Plata y muestras tomadas por el SAME Provincia (Servicio de emergencia del Estado Bonaerense). Para ello, se mantiene una continua comunicación con el equipo de coordinación de diagnóstico de COVID-19 perteneciente al Gabinete de Asesores del Ministerio de Salud de la Provincia de Buenos Aires, así como el Área de Epidemiología de la Región Sanitaria XI de la Provincia de Buenos Aires, para el armado de la logística que permite la correcta circulación de la muestra. Así, las muestras de hisopados nasofaríngeos son recolectadas por personal sanitario, colocadas en tubos con $2 \mathrm{ml}$ de solución fisiológica, dentro de bolsas herméticas y enviadas al laboratorio en un recipiente contenedor (triple envase), donde son recepcionadas junto con las fichas epidemiológicas correspondientes confeccionadas por el personal actuante en los muestreos. Los datos de las fichas epidemiológicas de cada muestra son cargados en el programa LabWin (http://www.biodatasa.com.ar/), y las muestras son procesadas con el número correlativo asignado por el programa. Los datos de procedencia (Municipios) y los datos clínicos son registrados para luego ser correlacionados con los resultados de los estudios moleculares. En virtud de estar 
Innovación y Desarrollo Tecnológico y Social (2020) 2 (2): 25-50- Número especial COVID-19

abocados, preferentemente, al programa DETeCTAr y teniendo en cuenta la necesidad de contar con los resultados en forma inmediata, los mismos son cargados en el SISA dentro de las 12/24 hs. de haber sido tomada la muestra. Además, se mantiene comunicación continua y directa con los profesionales y directivos de los diferentes establecimientos sanitarios.

Por otro lado, es importante destacar que recientemente se ha recibido financiamiento del MINCYT en el marco del proyecto “Detección y caracterización molecular del SARS-CoV-2 en animales y vigilancia epidemiológica de posibles reservorios, amplificadores y/o transmisores del virus" cuyo objetivo principal consiste en determinar datos epidemiológicos de animales en contacto con personas infectadas con SARS-CoV-2, y la detección y evaluación clínica de aquellos animales positivos, que permitan comprender la dinámica de la transmisión del virus y sus posibles reservorios, hospedadores intermediarios u otras vías de transmisión. Además, se espera poder realizar la caracterización genómica de las cepas circulantes y la obtención y conservación de muestras de interés epidemiológico para estudios posteriores (sangre, materia fecal, orina, etc.).

La proyección a futuro de este proyecto plantea la posibilidad de establecer un mecanismo de vigilancia epidemiológica de enfermedades virales y/o de otra índole en animales domésticos y fauna silvestre.

Cabe señalar además que a la fecha el grupo de trabajo ha publicado un trabajo científico denominado "Resultados del primer bimestre de trabajo de la Unidad de 
Diagnóstico COVID-19 de la Facultad de Ciencias Veterinarias-UNLP" en la revista Analecta Veterinaria (Moré et al, 2020), como asimismo ha realizado un informe sobre la comparación de 3 kits de real time RT-PCR para detección de SARS-CoV-2 enviado recientemente para su publicación.

\section{Aporte de la UNLP al sistema de salud de la Provincia de Buenos Aires}

El trabajo mancomunado de los laboratorios de las tres Facultades de la UNLP permitió no solo dar una respuesta temprana a la necesidad diagnóstica sino también significativa en cuanto a que se procesaron más de 32.000 muestras provenientes de Municipios de las Regiones Sanitarias I, II, IV, VI, X y XI de la Provincia de Buenos Aires (Figura 1).

En base a la experiencia adquirida durante el transcurso de la pandemia de SARSCoV-2 por parte de los laboratorios involucrados en este proyecto, la cual sienta la base de un trabajo conjunto de gran valor para la UNLP y una experiencia inigualable, consideramos pertinente la conformación de una "Red de Diagnóstico de Enfermedades Emergentes de Ia $\boldsymbol{U N}_{\mathbf{N}} \boldsymbol{P}^{\prime \prime}$, con el fin de amalgamar los conocimientos adquiridos y dejar funcionando un espacio permanente de apoyo al sistema sanitario provincial y nacional. 


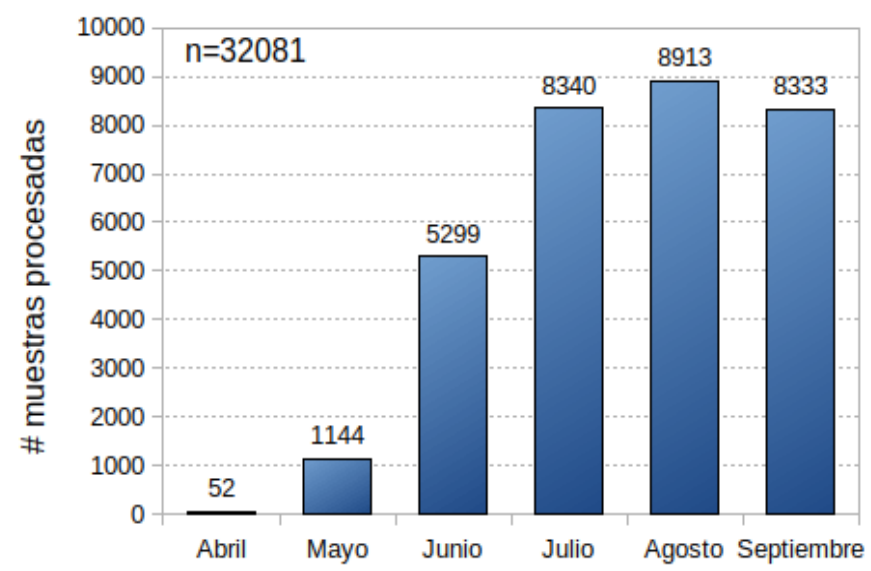

Figura 1. Número total de muestras procesadas por mes por los laboratorios de la FCE, FCM y FCV de la UNLP para la determinación del SARS-CoV-2.

Figure 1. Total number of samples processed per month by the laboratories of the FCE, FCM and FCV of the UNLP for the determination of SARS-CoV-2.

\section{Financiamiento}

En base a lo expresado en las cláusulas segunda y tercera del convenio específico (CONVE-2020-06345305-GDEBA-MSALGP), el Ministerio de Salud de la Provincia de Buenos Aires se compromete a suministrar los insumos necesarios para el diagnóstico molecular de COVID-19 y la Universidad Nacional de La Plata asegura las condiciones edilicias y de equipamiento correspondiente a los niveles de bioseguridad 2, cumpliendo con medidas de protección del personal recomendadas para este tipo de procedimientos.

En el caso del Laboratorio de Salud de la Facultad de Ciencias Exactas, parte de los fondos recibidos del MINCyT por el subsidio de Fortalecimiento de Capacidades Analíticas del LSP se destinan a la compra de equipamiento menor e insumos varios. 
También es importante resaltar que varios Institutos de Investigación y la facultad han destinado recursos para asegurar y sostener el funcionamiento del mismo.

\section{Agradecimientos}

Agradecemos al Presidente de la UNLP Dr. Fernando Tauber, a las autoridades de la Facultad de Ciencias Exactas, Ciencias Médicas, Ciencias Veterinarias de la UNLP, al Ministerio de Salud de La Provincia de Buenos Aires, así como al CONICET, CIC-PBA, Fundación Bioquímica Argentina y al Colegio de Bioquímicos de la Provincia de Buenos Aires.

\section{Referencias bibliográficas}

Corman, V. M., Landt, O., Kaiser, M., Molenkamp, R., Meijer, A., Chu, D. K., Bleicker, T., Brünink, S., Schneider, J., Schmidt, M. L., Mulders, D. G., Haagmans, B. L., van der Veer, B., van den Brink, S., Wijsman, L., Goderski, G., Romette, J. L., Ellis, J., Zambon, M., Peiris, M., ... Drosten, C. (2020). Detection of 2019 novel coronavirus (2019-nCoV) by real-time RT-PCR. Euro surveillance : bulletin Europeen sur les maladies transmissibles $=$ European communicable disease bulletin, 25(3), 2000045. https://doi.org/10.2807/1560-7917.ES.2020.25.3.2000045

Falleiros Arlant, L., de Colsa, A., Flores, D., Brea, J., Avila Aguero y Hozbor, D. (2014) Pertussis in Latin America: epidemiology and control strategies, Expert Review of Antiinfective Therapy, 12:10, 1265-1275. https://doi.org/10.1586/14787210.2014.948846 
Ministerio de Salud (2010). Coqueluche: normativa y tutorial para la vigilancia a través del sistema nacional de vigilancia laboratorial SIVILA-SNVS. https://www.snvs.msal.gov.ar/descargas/Instructivos Modulos/20100928 COQUEL

\section{$\underline{\text { UCHE Tutorial para la notificacion a traves del SIVILA 2010.pdf }}$}

Moré, G., Panei, C.J., Fuentealba, N., Aspitia, C., Bravi, M.E., Origlia, J., Rambeaud, M., Tizzano, M., Rudd Garcés, G., Golijow, C., Unzaga, J.M. y Pecoraro, M. (2020). Resultados del primer bimestre de trabajo de la unidad de diagnóstico COVID-19 de la Facultad de Ciencias Veterinarias-UNLP. Analecta Veterinaria, 40, 2, 050. https://doi.org/10.24215/15142590e050

Zhu, N., Zhang, D., Wang, W., Li, X., Yang, B., Song, J., Zhao, X., Huang, B., Shi, W., Lu, R., Niu, P., Zhan, F., Ma, X., Wang, D., Xu, W., Wu, G., Gao, G. F., Tan, W., \& China Novel Coronavirus Investigating and Research Team (2020). A Novel Coronavirus from Patients with Pneumonia in China, 2019. The New England journal of medicine, 382(8), 727-733. https://doi.org/10.1056/NEJMoa2001017

Provincia de Buenos Aires (2020). La provincia implementa un novedoso sistema de control

en geriátricos.

https://www.gba.gob.ar/saludprovincia/noticias/la provincia implementa un nove doso sistema de control en geri\%C3\%A1tricos

Wu, F., Zhao, S., Yu, B., Chen, Y.M., Wang, W., Song, Z.G., et al. A new coronavirus associated with human respiratory disease in China. Nature, 579, 265-269 (2020). https://doi.org/10.1038/s41586-020-2008-3 